\title{
Representing Ductile Damage with the Dual Domain Material Point Method
}

\author{
C. C. Long ${ }^{1}$, D. Z. Zhang ${ }^{1 *}$, C. A. Bronkhorst ${ }^{1}$, G. T. Gray III ${ }^{2}$ \\ ${ }^{1}$ Fluid Dynamics and Solid Mechanics, T-3 \\ Theoretical Division \\ ${ }^{2}$ Materials Science in Radiation and Dynamics Extreme, MST-8 \\ Materials Science and Technology Division \\ Los Alamos National Laboratory \\ Los Alamos, NM 87545
}

\begin{abstract}
In this paper, we incorporate a ductile damage material model into a computational framework based on the Dual Domain Material Point (DDMP) method. As an example, simulations of a flyer plate experiment involving ductile void growth and material failure are performed. The results are compared with experiments performed on high purity tantalum. We also compare the numerical results obtained from the DDMP method with those obtained from the traditional Material Point Method (MPM). Effects of an overstress model, artificial viscosity, and physical viscosity are investigated. Our results show that a physical bulk viscosity and overstress model are important in this impact and failure problem, while physical shear viscosity and artificial shock viscosity have negligible effects. A simple numerical procedure with guaranteed convergence is introduced to solve for the equilibrium plastic state from the ductile damage model.
\end{abstract}

Keywords: Material Point Method, Ductile Material Failure, Particle Methods

\section{Introduction}

Lagrangian finite element methods are the traditional choice to model solid deformations involving void growth and material failure. However, difficulties arise when the material experiences large deformation resulting in heavily skewed elements. To avoid this numerical difficulty, we use the dual domain material point

\footnotetext{
*Corresponding author, email: dzhang @lanl.gov
} 
(DDMP) method [1] for these types of problems. The DDMP method is an improved version of the material point method (MPM) [2], which is the particle-incell (PIC) method [3] reformulated using the concept of virtual work for historydependency in constitutive modeling. Since the concept of virtual work is also the starting point of the finite element method (FEM), many numerical properties are similar between the material point methods (both DDMP and MPM) and the FEM. The most significant difference between the material point methods and the FEM is that material point methods retain Lagrangian capabilities using an Eulerian mesh, rather than a Lagrangian mesh as in FEM. The Lagrangian capability of the material point method relies on the Lagrangian particles, also called material points, that are free to move about the Eulerian mesh, and thus can be used to carry the history dependent quantities in ductile material models without the difficulties of numerical diffusion. At each time step, data from the moving particles is transferred to the underlying grid where numerical analysis can be readily performed.

Material point methods avoid mesh distortion difficulties associated with Lagrangian methods and numerical diffusion difficulties associated with Eulerian methods because these methods use both an Eulerian mesh and Lagrangian particles. However, the MPM is far from problem free for cases involving large material deformations because the MPM uses gradients of shape functions at particle locations to calculate the nodal force. When a particle crosses a cell boundary, numerical noise is created since the gradient is discontinuous across cell boundaries [4]. Several schemes [4, 5] have been explored to avoid this numerical noise. All of the schemes introduce the concept of a particle domain, which is equivalent to modifying the shape functions in the MPM. To conserve mass and momentum, these particle domains are required to cover the entire domain occupied by the material without overlap or gap. A rigorous enforcement of this requirement brings back mesh distortion difficulties of the finite element method. In practical uses of these schemes, this requirement is relaxed $[4,5]$ by accepting errors in mass and momentum conservation.

The DDMP method [1] takes a different approach to avoid the numerical noise by modifying the gradient of the shape function to make it continuous across cell boundaries, without changing the shape function of the MPM. In this way the concept of particle domain is not needed. As a consequence, the influence of a particle is different for the momentum and force calculations. Therefore the method is named as the "dual domain" material point method. The method has been shown to rigorously conserve mass and momentum, while the error in energy conservation is second order in both temporal and spatial discretization. 
The DDMP method has been applied to many large deformation problems $[6,7]$ with success. In the present work we use the DDMP method to perform continuum mechanics calculations of a flyer plate experiment using a ductile damage model. The present work has three objectives. The first objective is to evaluate whether the DDMP method can represent the major physics involved in this problem, including plastic flow, porosity growth, material damage, and failure. The second objective is to understand the numerical properties of the DDMP method in materials with ductile damage and strain softening. The third objective is to use the numerical capability of DDMP to evaluate the relative importance of different physical mechanisms and numerical treatments often used in modeling this impact and failure problem. The noise reduction provided by the DDMP method is important in this flyer plate problem. In the region where the material is about to fail, the softening of the material leads to localized strain and large displacement of the particles causing them to move across cell boundaries.

The basis for the flyer plate problem is straightforward: A plate is traveling at high velocity and strikes another stationary plate. These are referred to as the flyer plate and the target plate, respectively. The target plate has a thickness double that of the flyer plate. Upon impact, a compression shock travels through both plates to opposite surfaces, and the reflected tensile expansion fans collide at the center of the target plate, if the flyer and the target plates are made of the same material. For a critical value of initial velocity of the flyer plate, voids are created in a typical ductile material leading to softening and eventually failure along the center line of the target plate as shown in Fig.1. This problem is physically well defined, while presenting numerous challenges for numerical techniques, including shock propagation, material softening and failure, and history dependence. Therefore we choose this problem to test the DDMP method. The DDMP method is described briefly in the next section. Readers interested in the method are encouraged to reference [1] for further details.

\section{Dual Domain Material Point Method}

As shown in Fig. 1, the target plate develops a highly porous region near the center line in the experiment. It is not practical to numerically consider the individual pores and cracks, and we employ the work of Addessio and Johnson [8, 9], who developed a ductile damage model based on the continuum description to capture the effects of void growth in aggregate. Our starting point is the contin- 


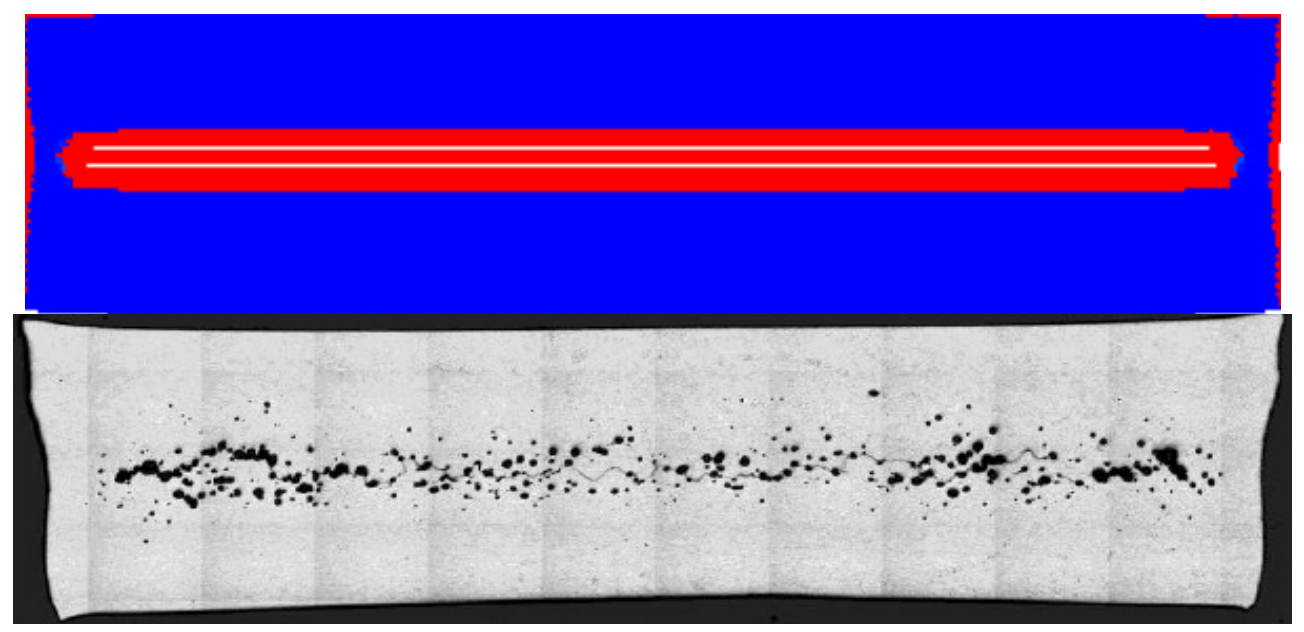

Figure 1: Plot of numerical porosity results (top) and experimental image (bottom). Numerical porosity is plotted, where blue is low porosity $(\Phi<0.001)$ and red is high porosity $\left(\Phi=\Phi_{f}=0.4\right)$.

uum description of the material. The momentum equation can be written as

$$
\rho \frac{d \mathbf{v}}{d t}=\nabla \cdot \sigma
$$

where $\rho$ is the density of the bulk material, $\mathbf{v}$ is the velocity, and $\sigma$ is the stress tensor, which depends on the history of the material deformation and growth of the voids.

To model the deformation and damage response of metallic materials, we use the DDMP method. As mentioned, the DDMP method uses Lagrangian particles, also called material points, in addition to an Eulerian grid, where the computational cells and nodal points are fixed in space. The Lagrangian particles carry information of material deformation, and naturally track the history of the material. Conservation quantities, such as mass and momentum are calculated on the Eulerian mesh nodes. For cases involving chemical reaction or heat and species diffusion and exchange among phases or species, in our code CartaBlanca, the internal energy and specie concentrations are also calculated on mesh nodes following the weak solution principle for the partial differential equations [10]. However, for the single material problem studied in the present paper, because of the short time scale in the material deformation, heat conduction can be neglected, and the process can be approximated as adiabatic. As an option in the code, we skip the Eulerian consideration of the internal energy field. The material temperature is then calculated in a Lagrangian manner following the particles. The Lagrangian 
particles are seeded initially in the Eulerian grid. Typical seeding method is to divide an Eulerian cell into smaller regions and then to place material points in the centers of these regions. The volume of the region is assigned to be the initial volume of the particle. The subsequent particle volume is updated each time step according to the velocity divergence at the particle location. For the twodimensional calculations performed in the present work, we use rectangular cells. To seed the particles, each cell is divided into $2 \times 2$ regions in the $x$ and $y$ directions. The material points are then placed in the centers of these regions. Therefore 4 particles per cell are used in the initial particle seeding. Our experiences show that $2 \times 2$ particles per cell are usually sufficient for DDMP calculations with a maximum strain less than $20 \%$, which is true for the present work. After initial seeding, these material points, or particles, are allowed to move following the motion of the material.

For more general cases, depending on local strains, particles can aggregate in some areas and disperse in others. If some region of the material experiences a large tensile strain, the particle distribution can become too scarce, and the numerical accuracy could suffer. For those cases we have successfully initialized using $3 \times 3$ and $4 \times 4$ particles per cell [1]. In principle, we can also dynamically allocate particles. For instance, particle splitting method has been used [11], in which a particle is split into two when its size is stretched to exceed a certain fraction of the cell size. This scheme has not been implemented in our current code partially because the use of the DDMP method, in which a particle has an influence region of two cells in the nodal force calculation.

The MPM uses the same shape functions for the given mesh as in the finite element method. The velocity at position $\boldsymbol{x}$ can be approximated by interpolating the surrounding nodal velocities to the position as

$$
\mathbf{v}(\boldsymbol{x}, t)=\sum_{i=1}^{N} \mathbf{v}_{i}(t) S_{i}(\boldsymbol{x})
$$

where $N$ is the number of grid points in the domain, $\mathbf{v}_{i}$ is the nodal velocity, and $S_{i}(\boldsymbol{x})$ is the shape function associated with node $i$. Using the same shape functions to approximate the virtual velocity, after the application of the principle of virtual work, one finds the discretized momentum equation [2]:

$$
m_{i} \frac{d \mathbf{v}_{i}}{d t}=-\sum_{p=1}^{N_{p}} v_{p} \boldsymbol{\sigma}_{p} \cdot \nabla S_{i}\left(\boldsymbol{x}_{p}\right)+\int_{\partial \Omega} \boldsymbol{\sigma} \cdot \boldsymbol{n} S_{i} d A,
$$




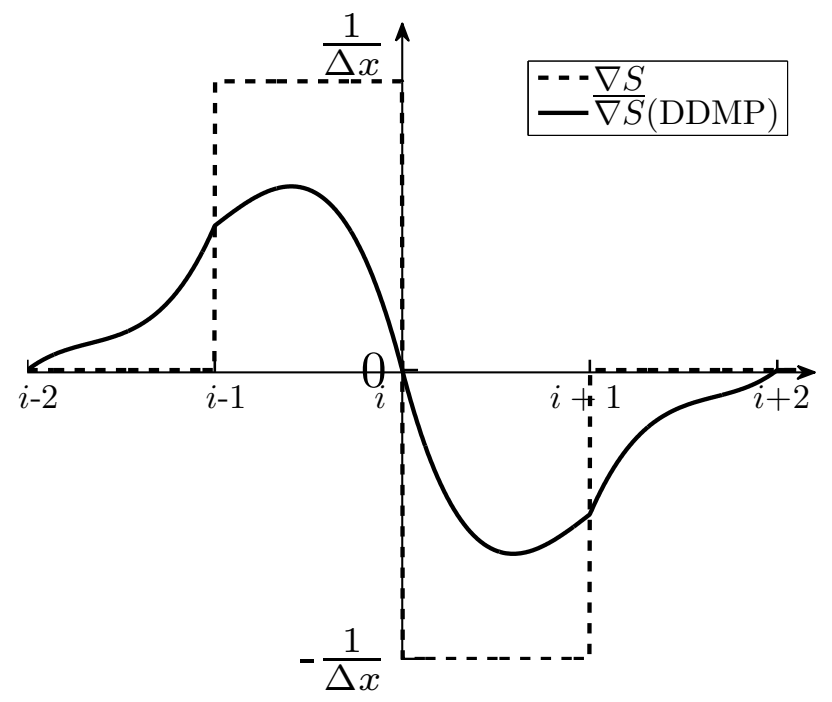

Figure 2: Illustration of the gradient of the shape function used in traditional MPM (dotted line) and used in DDMP (solid line) at node $i . \Delta x$ is the grid spacing.

where $m_{i}$ is a mass associated with node $i$ on the Eulerian grid, $\sigma_{p}$ is the stress at particle $p, v_{p}$ is the volume of the particle, and $N_{p}$ is the number of particles in the domain $\Omega$, which is chosen to contain large enough space for the material to move and deform. Different from a finite element calculation, in material point methods the computational domain can contain many empty cells. Although book keeping and computer memory are needed for these empty cells, the computational effort used for these cells is negligible [12], as the dominant computational cost in material point methods is updating particle state variables, volumes, and positions. To reduce the memory size, one can choose to allocate mesh cells dynamically as described in [13].

Equation 3 is used directly in the original MPM. A significant problem arises due to the discontinuity in the shape function gradient, $\nabla S_{i}\left(\boldsymbol{x}_{p}\right)$, which is illustrated for a one-dimensional case in Fig. 2. The discontinuity of the gradient at node $i$ causes the first term on the right of Eq. 3, called the internal force, to suddenly switch signs when a particle crosses a cell boundary, creating numerical noise and leading to possible instability.

To avoid this numerical noise, the DDMP uses identical shape functions as in the original MPM, but replaces its gradient $\nabla S_{i}$ in Eq. 3 with

$$
\overline{\nabla S_{i}}(\boldsymbol{x})=\alpha(\boldsymbol{x}) \nabla S_{i}(\boldsymbol{x})+[1-\alpha(\boldsymbol{x})] \widetilde{\nabla S_{i}}(\boldsymbol{x}),
$$


where

$$
\alpha(\boldsymbol{x})=0.5\left\{\prod_{k=1}^{n_{c}}\left[n_{c} S_{k}(\boldsymbol{x})\right]\right\}^{\frac{3}{2\left(n_{c}-1\right) d}}
$$

and

$$
\widetilde{\nabla S_{i}}(\boldsymbol{x})=\sum_{j=1}^{N} \frac{S_{j}(\boldsymbol{x})}{V_{j}} \int_{\Omega} S_{j} \nabla S_{i} d v,
$$

with $V_{j}$ being a control volume at node $j, n_{c}$ being the number of nodes in a computational cell, and $d$ being the number of spatial dimensions. This modified gradient of the shape function is illustrated in Fig. 2 for a one-dimensional case. Such a modified gradient of the shape function is continuous, although not smooth. Since the modified gradient of the shape function has a larger support (nonzero region) than that of the shape function, this method is called the dual domain material point (DDMP) method. Although the support of the modified gradient of a shape function is enlarged to include the immediate neighbor cells, all the non-local effects of a particle can be accounted for by two consecutive local operations. Therefore this scheme can be implemented in calculations with an unstructured mesh. The numerical instability caused by the discontinuity of $\nabla S_{i}$ is eliminated when $\overline{\nabla S_{i}}(\boldsymbol{x})$ replaces $\nabla S_{i}$. It is proven [1] that the modified gradient $\overline{\nabla S_{i}}(\boldsymbol{x})$ satisfies all properties sufficient [14] for the method to converge to the solution of the partial differential equation as the mesh and the time step are refined. Furthermore, for a given mesh, the approximate solution guarantees mass and momentum conservations and has a second-order accuracy in energy conservation with respect to spatial and time discretization.

Examples [1, 6, 7] have shown that the use of DDMP can eliminate the noise generated by cell crossing particles. After nodal accelerations are calculated from Eq. 3, the material point velocities are updated using particle accelerations, which are interpolated from the nodal accelerations to the particle locations as described by the fluid-implicit-particle method (FLIP) [15]. Instead of directly interpolating the node velocities to particles, this scheme for particle velocity update ensures the particle-in-cell (PIC) [3] family of methods (including MPM and DDMP) have very small (sometimes even regarded as zero) numerical diffusion. This is because the calculated particle velocity will not change unless the right hand side of Eq. 3 is nonzero. In other words, all the change in the particle velocity is caused by the physical driving force represented by the right hand side of Eq. 3, and not for any numerical reason. 


\section{Material model}

In the present work we implement the ductile damage and failure model of Addessio and Johnson as described in [8, 9]. Early implementations [8] used a constant plastic flow stress. Following [16], in the present work we use a Gurson surface to define deformation in the plastic regime. An implicit implementation which includes an overstress model is also derived from [16].

We use the Mie-Gruneisen equation of state:

$$
\begin{aligned}
\mu & =\frac{\rho_{s}}{\rho_{s_{0}}}-1, \\
P_{s} & =B_{s} \mu\left(1-\frac{\Gamma_{s} \mu}{2}\right)+\Gamma_{s} e_{s} \rho_{s},
\end{aligned}
$$

where subscript $s$ denotes the solid matrix (excluding voids), $P_{s}$ is the average pressure, $B_{s}$ is the bulk modulus, $\Gamma_{s}$ is the Gruneisen coefficient, $e_{s}$ is the internal energy, $\rho_{s_{0}}$ is the initial solid density, and $\mu$ is the compression ratio.

The mixture pressure $P$, bulk modulus $B$ and shear modulus $G$ are reduced from their corresponding matrix values because of the presence of the porosity $\Phi$ as [17]

$$
P=P_{s}(1-\Phi), \quad B=B_{s}(1-\Phi), \quad G=G_{s}(1-\Phi) .
$$

Since the voids do not contain any energy, the internal energy is not reduced because of the presence of the voids, and

$$
e=e_{s}
$$

Following the work of $[8,9]$, the porosity growth is associated with the rate of plastic volumetric deformation, $\dot{\varepsilon}_{p_{k k}}$,

$$
\dot{\Phi}=(1-\Phi) \dot{\varepsilon}_{p_{k k}} \text {. }
$$

The plastic deformation rate is calculated according to the plastic flow rule described in the next section.

Using these relations, the evolution equations for the pressure and deviatoric stress $\boldsymbol{s}$ of the bulk material are obtained [16].

$$
\begin{aligned}
& \dot{P}=-B \dot{\varepsilon}_{k k}+\alpha \dot{\boldsymbol{\varepsilon}}_{p_{k k}}+\Gamma_{s} \boldsymbol{s}: \dot{\boldsymbol{e}}, \\
& \dot{\boldsymbol{s}}=(\boldsymbol{\omega} \boldsymbol{s}-\boldsymbol{s} \boldsymbol{\omega})+2 G\left(\dot{\boldsymbol{e}}-\dot{\boldsymbol{e}}_{p}\right),
\end{aligned}
$$

where $\alpha=B-\left(1+\Gamma_{s}\right) P, \omega$ is the vorticity tensor, $\dot{\boldsymbol{e}}$ is the deviatoric strain rate tensor, and $\dot{\boldsymbol{e}}_{p}$ is the deviatoric plastic strain rate tensor. Equations 12 and 13 are integrated numerically with the plastic flow models described in the following section. 


\section{Plastic flow, material damage, and failure}

To numerically integrate equations 12 and 13 we write them in incremental forms

$$
\begin{gathered}
P^{n+1}=P^{t}+\alpha \Delta \varepsilon_{p_{k k}}, \\
\boldsymbol{s}^{n+1}=\boldsymbol{s}^{t}-2 G \Delta \boldsymbol{e}_{p},
\end{gathered}
$$

where $\Delta \varepsilon_{p_{k k}}=\dot{\varepsilon}_{p_{k k}} \Delta t$ and $\Delta \boldsymbol{e}_{p}=\dot{\boldsymbol{e}}_{p} \Delta t$ are the volumetric and deviatoric plastic strain increments over the time step $\Delta t$, and

$$
\begin{aligned}
P^{t} & =P^{n}-B \dot{\varepsilon}_{k k}^{n+1} \Delta t+\Gamma_{s} \boldsymbol{s}^{n}: \dot{\boldsymbol{e}}^{n+1} \Delta t, \\
\boldsymbol{s}^{t} & =\boldsymbol{s}^{n}+\Delta t\left(\boldsymbol{\omega} \boldsymbol{s}^{n}-\boldsymbol{s}^{n} \boldsymbol{\omega}\right)+2 G \Delta t \dot{\boldsymbol{e}} .
\end{aligned}
$$

Pressure $P^{t}$ and $\boldsymbol{s}^{t}$ are called the trial state pressure and deviatoric stress. As defined in Eqs. 16 and 17, the trial state is calculated assuming no plastic deformation during the time step.

\subsection{Equilibrium state}

To calculate the plastic strain in a time step, we first find the equilibrium state, which is related to equivalent stress, equivalent plastic strain, plastic flow stress, and the Gurson function defined as follows.

For a given deviatoric stress tensor $\boldsymbol{s}$ and a plastic strain tensor $\boldsymbol{\varepsilon}_{p}$, the equivalent stress $\tau$ and equivalent plastic strain $\bar{\varepsilon}_{p}$ are defined as

$$
\tau=\sqrt{\frac{3}{2} s: s}, \quad \bar{\varepsilon}_{p}=\sqrt{\frac{2}{3} \varepsilon_{p}: \varepsilon_{p}} .
$$

The plastic flow stress $\sigma_{s}$ [16] is obtained from the Johnson-Cook model [18] using these definitions of equivalent stress and equivalent plastic strain,

$$
\sigma_{s}=\left[A_{1}+A_{2}\left(\bar{\varepsilon}_{p}\right)^{n}\right]\left\{1+A_{3} \ln \left[\max \left(\frac{\dot{\bar{\varepsilon}}_{p}}{\dot{\varepsilon}_{0}}, 1\right)\right]\right\}\left[1-\left(\frac{T-T_{0}}{T_{m}-T_{0}}\right)^{m}\right],
$$

where $A_{i}, n$, and $m$ are material constants provided in Table $1, \dot{\bar{\varepsilon}}_{p}$ is the equivalent plastic strain rate, $\dot{\varepsilon}_{0}=1 \mathrm{~s}^{-1}$ is a reference plastic strain rate, and $T, T_{m}$, and $T_{0}$ are the current, melting, and reference temperatures of the material, respectively. Using these quantities the Gurson function [19] is defined as:

$$
F(\tau, \Phi, P)=\left(\frac{\tau}{\sigma_{s}}\right)^{2}-\left(1+q_{3} \Phi^{2}-2 q_{1} \Phi \cosh (\beta)\right),
$$


where $\beta=-3 q_{2} P /\left(2 Y_{f}\right)$, with $Y_{f}$ being the saturated stress limit of the solid matrix, and $q_{1}, q_{2}$, and $q_{3}$ are material constants found in Table 1.

To find the equilibrium state, we use the trial state values of $P, \Phi, \boldsymbol{s}, \boldsymbol{\varepsilon}_{p}$, and $\dot{\bar{\varepsilon}}_{p}$ in Eqs. 18, 19 and 20, to compute $F\left(\tau^{t}, \Phi^{t}, P^{t}\right)$. Since the trial state by definition is a state with no increment in plastic strain, we can write the plastic strains and porosity as $\boldsymbol{\varepsilon}_{p}^{t}=\boldsymbol{\varepsilon}_{p}^{n}, \dot{\varepsilon}_{p}=0$, and $\Phi^{t}=\Phi^{n}$, respectively, where the superscript $n$ denotes the previous time step values. If $F\left(\tau^{t}, \Phi^{t}, P^{t}\right) \leq 0$, then the deformation is purely elastic in this time step, $\dot{\boldsymbol{\varepsilon}}_{p}^{e}=\mathbf{0}$, and the trial state is accepted as the updated material state at time $n+1$. However, if $F\left(\tau^{t}, \Phi^{t}, P^{t}\right)>0$, then plastic deformation and porosity change occurs. The equilibrium state satisfies $F\left(\tau^{e}, \Phi^{e}, P^{e}\right)=0$, where the superscript $e$ denotes the equilibrium state. The plastic strain rate in the equilibrium state is assumed to be normal to the Gurson surface, or equivalently, the plastic strain tensor can be scaled by a Lagrangian multiplier, $\Delta \lambda$, as

$$
\Delta \boldsymbol{\varepsilon}_{p}^{e}=\Delta \lambda \frac{\partial F}{\partial \boldsymbol{\sigma}^{e}}
$$

By differentiating Eq. 20, this relation can be written in terms of the volumetric and the deviatoric components,

$$
\begin{aligned}
\Delta \varepsilon_{p_{k k}}^{e} & =\Delta \lambda \frac{3 q_{1} q_{2}}{Y_{f}} \Phi^{e} \sinh \left(\beta^{e}\right), \\
\Delta \boldsymbol{e}_{p}^{e} & =\Delta \lambda \frac{3}{\sigma_{s}^{2}} \boldsymbol{s}^{e} .
\end{aligned}
$$

Substitution of Eqs. 22- 23 into Eqs. 11, 14, 15, and requiring that the equilibrium state is on the Gurson surface yield the following four equations:

$$
\begin{aligned}
\boldsymbol{s}^{e} & =\boldsymbol{s}^{t}-2 G \dot{\boldsymbol{e}}_{p}^{e} \Delta t=\boldsymbol{s}^{t}\left(\frac{1}{1+\frac{6 G \Delta \lambda}{\sigma_{s}^{2}}}\right), \\
P^{e} & =P^{t}+\alpha \Delta \lambda \frac{3 q_{1} q_{2}}{Y_{f}} \Phi^{e} \sinh \left(\beta^{e}\right), \\
\Phi^{e} & =\Phi^{t}+\left(1-\Phi^{e}\right) \Delta \lambda \frac{3 q_{1} q_{2}}{Y_{f}} \Phi^{e} \sinh \left(\beta^{e}\right), \\
F\left(\tau^{e}, \Phi^{e}, P^{e}\right) & =\left(\frac{\tau^{e}}{\sigma_{s}}\right)^{2}-\left[1+q_{3} \Phi^{e 2}-2 q_{1} \Phi^{e} \cosh \left(\beta^{e}\right)\right]=0 .
\end{aligned}
$$

The equilibrium material state is found from the trial states by solving for a new porosity $\Phi^{e}$, pressure $P^{e}$, plastic multiplier $\Delta \lambda$, and deviatoric stress $\boldsymbol{s}^{e}$ 
from these equations. These equations make up what is sometimes referred to as the Tensile Plasticity (TEPLA) model. There are explicit [9] and implicit [16] approaches of solving these coupled equations. The explicit approach may require smaller time steps due to the equilibrium solutions being projected to the interior of the Gurson surface, and requires a numerical check to ensure this is not the case. The typical implicit approach uses Newton iterations [16] to solve the four equations simultaneously. Convergence may pose problems if the trial state is not sufficiently close to the solution.

We introduce a simpler method of solving the four coupled nonlinear equations, in which the first three equations (24 - 26) are solved analytically, and the fourth equation is solved using the bisection method to guarantee convergence. We begin by rewriting Eq. 26 as

$$
\Delta \lambda \frac{3 q_{1} q_{2}}{Y_{f}} \Phi^{e} \sinh \left(\beta^{e}\right)=\frac{\Phi^{e}-\Phi^{t}}{1-\Phi^{e}} .
$$

Substituting this into Eq. 25, and noting $\alpha=B-\left(1+\Gamma_{s}\right) P^{e}$, we solve for $P^{e}$ and find

$$
P^{e}=\frac{P^{t}+B \frac{\Phi^{e}-\Phi^{t}}{1-\Phi^{e}}}{1+\left(1+\Gamma_{s}\right) \frac{\Phi^{e}-\Phi^{t}}{1-\Phi^{e}}} .
$$

With such obtained $P^{e}$, we calculate $\beta^{e}=-\frac{3 P^{e} q_{2}}{2 Y_{f}}$, and use it to calculate $\Delta \lambda$ from Eq. 25,

$$
\Delta \lambda=\frac{Y_{f}\left(P^{e}-P^{t}\right)}{3 \alpha q_{1} q_{2} \Phi^{e} \sinh \left(\beta^{e}\right)} .
$$

With this $\Delta \lambda$, the deviatoric stress $\boldsymbol{s}^{e}$ can be easily obtained using Eq. 24 and then the equilibrium equivalent stress $\tau^{e}$ can also be calculated from Eq. 18. In this way, with the given trial state, the equilibrium pressure $P^{e}$, the plastic multiplier $\Delta \lambda$, and the equivalent stress $\tau^{e}$ can be calculated directly from porosity $\Phi^{e}$. In other words, for a given $\Phi^{e}$, we have solved $P^{e}, \Delta \lambda$, and $\tau^{e}$ analytically from Eqs. 24, 25 and 26. After substituting these values into Eq. 27, the Gurson function $F\left(\tau^{e}, \Phi^{e}, P^{e}\right)$ is reduced to a single variable function of $\Phi^{e}$. In this process we analytically reduce four coupled nonlinear equations to a single nonlinear equation in terms of porosity $\Phi^{e}$. A bisection method is then used to solve for the porosity $\Phi^{e}$ from Eq. 27 , with guaranteed convergence. In the bisection method we also take advantage of the fact that $\Phi^{e}$ is bounded by $\Phi_{0}$ and $\Phi_{f}$, the initial and failure porosity.

To accelerate the iteration process, we further constrain the bounds for the equilibrium porosity. If the material is in tension, the porosity can only grow, and 
the bound for $\Phi^{e}$ is then from $\Phi^{t}$ to $\Phi_{f}$, as $\Phi^{t}$ is equilibrium porosity in the last time step. For a material in compression, the porosity can only reduce, and the bound is then from $\Phi_{0}$ to $\Phi^{t}$. The iteration is continued until convergence. A brief study on the appropriate threshold value for convergence tolerance is reported in Sec. 5. After convergence, the Cauchy stress is set by: $\boldsymbol{\sigma}^{e}=-P^{e} \mathbf{I}+\boldsymbol{s}^{e}$, and the plastic deformation tensor is updated as:

$$
\boldsymbol{\varepsilon}_{p}^{n+1}=\boldsymbol{\varepsilon}_{p}^{n}+\Delta \boldsymbol{e}_{p}+\Delta \varepsilon_{p_{k k}} \boldsymbol{I}
$$

where

$$
\begin{gathered}
\Delta \boldsymbol{e}_{p}=\Delta \lambda \frac{3 \boldsymbol{s}^{e}}{\sigma_{s}^{2}}=\frac{\boldsymbol{s}^{t}-\boldsymbol{s}^{e}}{2 G} \\
\Delta \varepsilon_{p_{k k}}=\Delta \lambda \frac{3 q_{1} q_{2}}{Y_{f}} \Phi^{e} \sinh \left(\beta^{e}\right)=-\frac{P^{t}-P^{e}}{\alpha}
\end{gathered}
$$

if the overstress model is not used. If the overstress model is used, $\Delta \boldsymbol{e}_{p}$ and $\Delta \varepsilon_{p_{k k}}$ are calculated according to the following subsection.

\subsection{Overstress}

With an overstress model, Addessio and Johnson [9], and Zuo and Rice [16] introduced a porosity dependent overstress viscosity,

$$
\mu_{O S}=\eta\left(\frac{1-\Phi_{0}}{\Phi_{0}}\right)^{\frac{2}{3}}\left(\frac{1-\Phi}{\Phi}\right)^{\frac{1}{3}}
$$

where $\eta$ is a user-defined variable with units of viscosity. They assume that the rate of plastic deformation is proportional to the difference between the current stress state and the equilibrium state, as shown:

$$
\dot{\boldsymbol{\varepsilon}}_{p}=\frac{1}{3 \mu_{O S}}\left(\boldsymbol{\sigma}^{n+1}-\boldsymbol{\sigma}^{e}\right)
$$

Written in component form, this equation becomes

$$
\begin{aligned}
\dot{\varepsilon}_{p_{k k}} & =\frac{-1}{\mu_{O S}}\left(P^{n+1}-P^{e}\right) \\
\dot{\boldsymbol{e}}_{p} & =\frac{1}{3 \mu_{O S}}\left(\boldsymbol{s}^{n+1}-\boldsymbol{s}^{e}\right) .
\end{aligned}
$$

Instead of assuming the deformation to the equilibrium state is completed in one time step, overstress model introduces relaxation times $\xi=\mu_{O S} / \alpha$ for the pressure 
and $\zeta=3 \mu_{O S} /(2 G)$ for the deviatoric stress. With these plastic strain rates, Eqs. 12 and 13 become

$$
\begin{aligned}
\dot{P} & =-B \dot{\varepsilon}_{k k}+\Gamma_{s} \boldsymbol{s}: \dot{\boldsymbol{e}}-\frac{\alpha}{\mu_{O S}}\left(P-P^{e}\right), \\
\dot{\boldsymbol{s}} & =2 G \dot{\boldsymbol{e}}-(\boldsymbol{s} \boldsymbol{\omega}-\boldsymbol{\omega} \boldsymbol{s})-\frac{2 G}{3 \mu_{O S}}\left(\boldsymbol{s}-\boldsymbol{s}^{e}\right),
\end{aligned}
$$

where $P^{e}$ and $\boldsymbol{s}^{e}$ are the equilibrium pressure and deviatoric stress found in the previous subsection. An implicit discretization of these equations leads to [16]

$$
\begin{aligned}
P^{n+1} & =\frac{\frac{\xi}{\Delta t} P^{t}+P^{e}}{1+\frac{\xi}{\Delta t}}, \\
\boldsymbol{s}^{n+1} & =\frac{\frac{\zeta}{\Delta t} \boldsymbol{s}^{t}+\boldsymbol{s}^{e}}{1+\frac{\zeta}{\Delta t}}
\end{aligned}
$$

where $P^{t}$ and $\boldsymbol{s}^{t}$ are the trial pressure and deviatoric stress defined in Eqs. 16 and 17. Using Eqs. 41 and 40 in Eqs. 36 and 37, we find

$$
\begin{aligned}
\Delta \varepsilon_{p_{k k}} & =-\frac{P^{t}-P^{e}}{\alpha\left(1+\frac{\xi}{\Delta t}\right)}, \\
\Delta \boldsymbol{e}_{p} & =\frac{\boldsymbol{s}^{t}-\boldsymbol{s}^{e}}{2 G\left(1+\frac{\zeta}{\Delta t}\right)} .
\end{aligned}
$$

Substitution of Eq. 42 into Eq. 11 leads to

$$
\Phi^{n+1}=\frac{\Phi^{n}+\Delta \varepsilon_{p_{k k}}^{n+1}}{1+\Delta \varepsilon_{p k}^{n+1}} .
$$

Equations 41 and 40 show that with this overstress model, the pressure and the deviatoric stress at the end of a time step are weighted averages between the trial values and the equilibrium values. Thus, for a small value of $\mu_{O S}$, the relaxation times are also small, and the pressure, deviatoric stress, plastic strains, and porosity approach the corresponding equilibrium values found in the previous section. Contrariwise, for very large values of $\mu_{O S}$, the final state approaches the trial state. This overstress viscosity $\mu_{O S}$ leads to a velocity diffusion and a length scale that provides the numerical stability after material failure. 


\subsection{Material Failure}

A Hancock-Mackenzie formulation is used to describe material failure in terms of the plastic strain and porosity [20]. The failure limit on plastic strain $\gamma_{f}$ is calculated from

$$
\gamma_{f}=\gamma_{0}+\gamma_{1} e^{\gamma_{2} \frac{P}{\tau}}
$$

where $\gamma_{0}, \gamma_{1}$, and $\gamma_{2}$ are material constants found in Table 1, $P$ is the pressure, and $\tau$ is the equivalent stress as defined in Eq. 18. A material particle is irredeemably 'failed' if

$$
\left(\frac{\Phi}{\Phi_{f}}\right)^{2}+\left(\frac{\varepsilon_{p}}{\gamma_{f}}\right)^{2} \geq 1,
$$

where $\Phi_{f}$ is a maximum value of porosity, and $\gamma_{f}$ is the equivalent plastic strain. When a material point fails by Eq. 46, the yield stress is set to zero.

\subsection{Artificial viscosity}

Artificial viscosity is a classical concept, first introduced by von Neumann and Richtmyer [21] as an additional pressure to a gas under compression to reduce numerical noise in the calculation of shocks. The additional pressure is traditionally computed as

$$
c_{q} \rho \ell^{2}\left(\frac{\partial \dot{x}}{\partial x}\right)^{2}-c_{L} \rho \ell a\left(\frac{\partial \dot{x}}{\partial x}\right),
$$

where, $c_{q}$ and $c_{L}$ are user-chosen coefficients, $\ell$ is a characteristic length scale, $\rho$ is the density of the medium, and $a$ is the speed of sound in the medium. The original formulation of $q$ was a quadratic diffusion-like quantity, the first term in Eq. 47. A linear (second) term was added by Landshoff in 1955 [22] to arrive at the traditional linear plus quadratic formulation that is commonly used today. Further work expanding the use of artificial viscosity to elastic solids and multiple dimensions has also been achieved, and we follow the work of [23, 24, 25, 26] to implement an artificial viscosity into our numerical code. In tensor form, the additional stress due to the artificial viscosity is

$$
\begin{aligned}
q_{i j} & =\sum_{k=1}^{d} \rho \ell Q_{i j_{k}}\left(\tau_{0} c_{L} a-c_{q} \ell \lambda_{k}\right), \\
\mathbf{Q}_{k} & =\lambda_{k} \mathbf{g}_{k} \mathbf{g}_{k}^{T},
\end{aligned}
$$

where $\mathbf{g}_{k}$ are the unity length eigenvectors of the strain rate tensor, $d$ is the number of spatial dimensions, $\lambda_{k}$ are the respective negative eigenvalues of the strain rate 
tensor $\left(\lambda_{k}=0\right.$, if the eigenvalue is positive), and

$$
\tau_{0}=\frac{|\nabla \cdot \mathbf{v}|}{\|\nabla \mathbf{v}\|_{2}}
$$

This term was introduced in [27], and was further explored in [26]. It has the effect of shrinking the contribution of the linear viscous term in the presence of high vorticity, which may occur locally. In the present work, we choose [28, 29] $c_{q}=2, c_{L}=0.5$ for all simulations presented unless otherwise noted. In the particle based calculation, the length scale $\ell$ is chosen to be the characteristic particle size, $\ell=\sqrt[3]{6 m_{p} / \rho}$, where $m_{p}$ is the mass of the particle, and $\rho$ is the current material density. With this calculated artificial stress, $\boldsymbol{q}$, the second term on the right of Eq. 3 is replaced with $\sigma_{p}+\boldsymbol{q}$ in the calculation of the acceleration.

\subsection{Solid Material Viscosity}

Shock-loaded metals have been modeled as highly viscous fluids [30] in which the energy dissipation is modeled as if the material were a Newtonian fluid. This method can be justified if the plastic deformation overwhelms the elastic deformation. Although for the cases considered in this work this condition is not satisfied, the concept of viscosity does bring in an interesting point. In traditional plasticity models for metals, the compressibility of the material is usually neglected, while a bulk volumetric deformation is allowed in $[8,9,16]$ and in the present work. To consider the energy dissipation associated with the volumetric deformation during a plastic flow, we explore the effect of the bulk viscosity $\mu_{B V}$ such that another viscous stress is added to the stress $\sigma_{p}$ in Eq. 3 as

$$
\boldsymbol{\sigma}=\boldsymbol{\sigma}_{p}+\mu_{B V}(\nabla \cdot \mathbf{v}) \boldsymbol{I}
$$

if the material is in a plastic state. In fact we have also experimented adding a shear viscosity term in the equation above. Similar to the finding reported in [7], we find that shear viscosity effects are negligible because the energy associated with deviatoric deformation has been sufficiently dissipated by the plastic flow. However, the bulk viscosity remains important as shown by the numerical examples in the next section. This is because the kinetic energy associated with the volumetric deformation is not dissipated elsewhere in this model. Although the bulk viscosity is in a similar form as the linear term of the artificial viscosity in Eq. 48, they are applied differently. The artificial viscosity is applied only to the compression strain rate regardless of the elastic or plastic states of the material. The bulk viscosity applies only to the material in the plastic state independent of 
the tension or compression strain rate. In the event of a plastic flow in compression, both bulk viscosity and artificial viscosity are active simultaneously, with the bulk viscosity largely dominating in magnitude.

The stress caused by this viscosity and the overstress contribute to the momentum equation, which determines size of the failure zone. Given an impact velocity $V$, using the momentum equation one can estimate [31] that the maximum tensile stress to be $\rho_{s 0} a V$ when the two tension waves superpose, where $a$ is the speed of tension wave. For tantalum in the elastic state $a \approx 3.3 \mathrm{~km} / \mathrm{s}$. The difference between this tensile stress and the equilibrium stress is balanced by the stress from this bulk viscosity and the overstress. Both these stresses are proportional to the strain rate. The length scale from the ratio between the impact velocity and this strain rate determines the size of the failure zone.

\section{Results}

Flyer plate experiments performed at Los Alamos National Laboratory [32] used metal plates of pure tantalum, with an initial flyer plate velocity of $249 \mathrm{~m} / \mathrm{s}$. The experimental and numerical setup is illustrated in Fig. 3. The velocity of the target plate is measured on the bottom surface. In the present work, we compare this measured velocity with numerical results. Additionally, we compare the calculated porosity field with the metallography images taken from the cross-section of the soft-captured samples of the target plate.

\subsection{Numerical Setup}

For all simulations presented in the present work, we initialize a computational domain measuring $4.5 \mathrm{~cm}$ by $1.5 \mathrm{~cm}$, as shown in Fig. 3. A flyer plate, represented by the blue particles in the figure, of dimensions $3.5 \mathrm{~cm}$ by $0.225 \mathrm{~cm}$ is initialized with a uniform velocity of $-249 \mathrm{~m} / \mathrm{s}$. The target plate has dimensions of $3.5 \mathrm{~cm}$ by $0.45 \mathrm{~cm}$, with vertical gaps positioned at $x=1.25$ and $x=3.25$, creating three distinct blocks. The gaps are placed to match experiments, with the center block being the target section of interest. All three pieces of the target plate are initially at rest. In this setup, the material of interest does not fill up the entire computational domain, which is a feature of using material point methods. The empty cells provide room for expected large material displacements or deformations. The use of empty cells requires only slight computational cost compared to filled ones, as most of the cost in the DDMP method is in the evaluation of the material points.

Initially, the porosity of all three target plates is assumed to be at the minimum, $\Phi=3.0 \times 10^{-4}$ as suggested in [9]. If the porosity falls below this value, we reset 


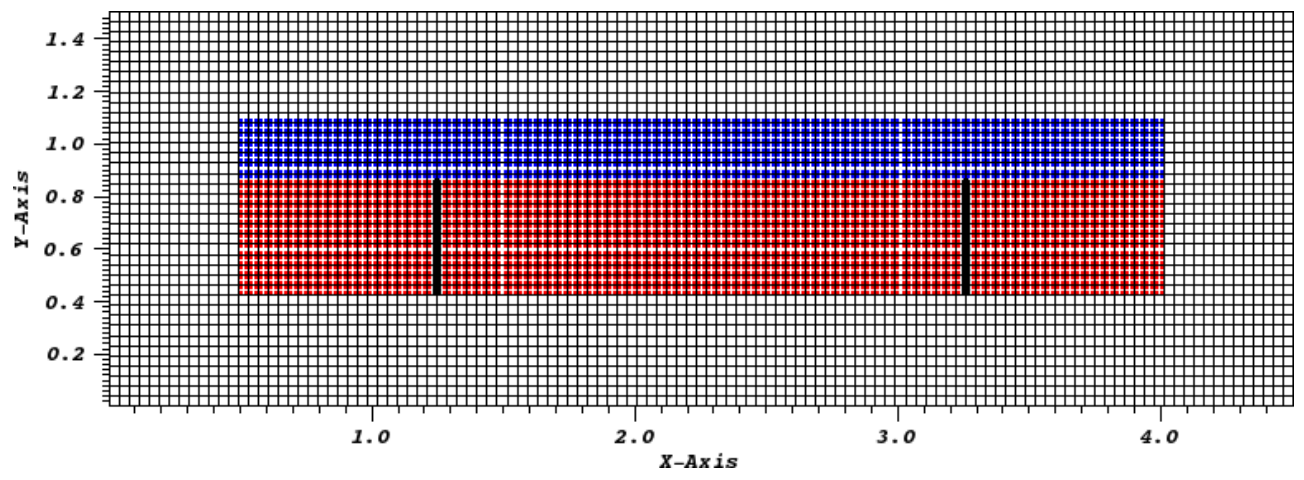

Figure 3: Plot of initial grid and particle positions. Blue indicates a flyer plate particle, and red is a target plate particle. Black vertical lines indicate crack locations.

it to this value and treat any plastic flow at this level as classic plastic flow under the Johnson-Cook model.

Impact occurs at $t=0$, and the simulation is run for $7 \mu$ s of physical time. Adaptive time stepping is utilized to ensure the CFL conditions are met on any given time step. All simulations are run serially, taking anywhere from 1 hour to two days to compute, depending on mesh resolution.

Initial investigations using artificial viscosity, bulk viscosity, and the overstress model produced good results compared with experimental velocity measurements, as well as experimental void formation. The results shown in Figs. 1 and 4 were generated using artificial viscosity in compression, an overstress term of $\eta=0.1$ $\mathrm{Pa} \cdot \mathrm{s}$, and a bulk viscosity defined as:

$$
\mu_{B V}= \begin{cases}0, & \text { if elastic deformation } \\ 10000 \mathrm{~Pa} \cdot \mathrm{s}, & \text { otherwise }\end{cases}
$$

The simulation was run using an underlying regular grid spacing of $150 \mu \mathrm{m}$.

\subsection{Material Model Convergence}

As discussed in Sec. 3, an iterative bisection method is used to solve the nonlinear Gurson equation 27 for the equilibrium plastic deformation in each time step. To ensure the convergence of our numerical results, we perform several simulations using a different convergent tolerance on the equation. The tightest tolerance used was $\left|F\left(\tau^{e}, \Phi^{e}, P^{e}\right)\right|<10^{-7}$. This case is referred to as the base case. We then compute several cases using larger tolerances, and we compare the resultant velocity field of each simulation to the base case. The velocity field error is 


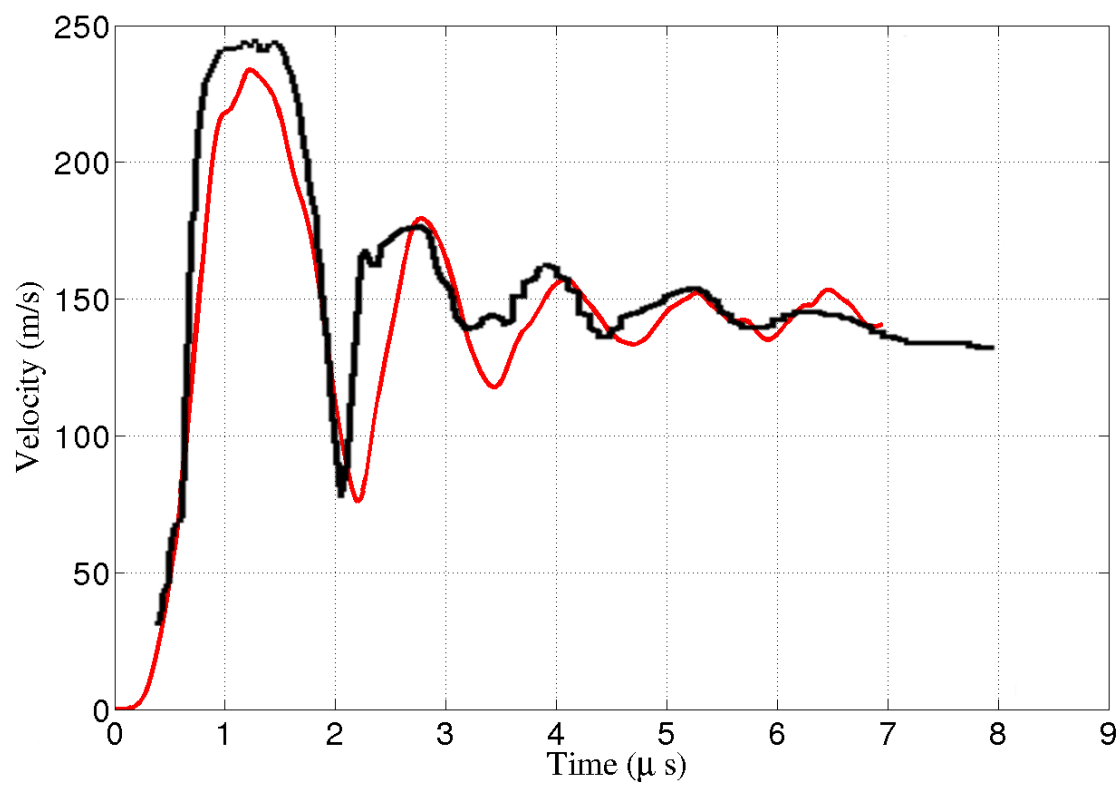

Figure 4: Plot of target plate velocity from both experimental (black) and numerical (red) results.

defined as follows:

$$
E=\left(\frac{\sum_{p=1}^{N_{p}}\left(\boldsymbol{v}_{p}-\boldsymbol{v}_{p b}\right)^{2}}{\sum_{p=1}^{N_{p}} \boldsymbol{v}_{p b}{ }^{\frac{1}{2}}},\right.
$$

where $\boldsymbol{v}_{p}$ is the velocity of particle $p$, the subscript $b$ designates the base case value, and $N_{p}$ is the number of particles in the simulation. This error is plotted in Fig. 5 as a function of time, and shows reasonable convergence for the Gurson surface tolerance.

Additionally, we desire to show that the method overall converges under mesh refinement. For this purpose, several simulations were run using coarser mesh resolutions with $2 \times 2$ particles per cell in the initial particle placement. These results are plotted in Fig. 6. In Figs. 7 and 8, we plot the effective plastic strain and the porosity calculated using different mesh resolutions.

After this mesh convergence study, the simulations reported in the rest of this section use a regular grid spacing of $150 \mu \mathrm{m}$. 


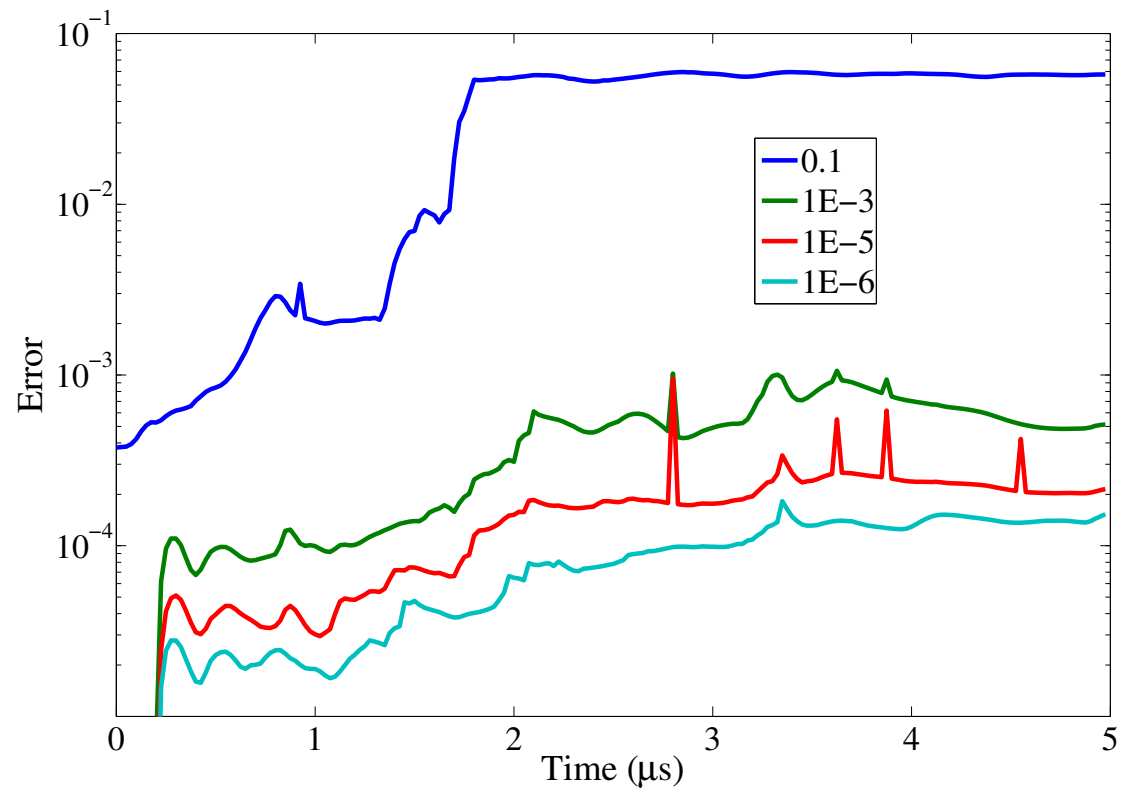

Figure 5: The velocity error, as defined in Eq. 53, for several tolerances within the iterative solver.

\subsection{Effects of Artificial and Bulk Viscosity}

Investigations into the effects of artificial and bulk viscosity was performed in the present work. Results from three simulations can be seen in Fig. 9. The blue line is calculated using bulk viscosity, artificial viscosity, and the overstress model. The simulation without bulk viscosity (green line) compares poorly with the experiment as shown in the figure. An improvement can be found by dramatically increasing the artificial viscosity coefficients, and by using the artificial viscosity terms in tension as well as compression, however this necessity suggests that a physical viscosity is missing from the system. The red line is computed using bulk viscosity only, without artificial viscosity or an overstress model. The bulk viscosity is $10000 \mathrm{~Pa} \cdot \mathrm{s}$, and acts only during plastic flow. The fact that the red line reproduces experimental data reasonably well, but not as good as the blue line suggest that both the overstress model and bulk viscosity are needed to model the flyer plate problem.

Artificial viscosity has long been used to provide dissipation to noise near discontinuities in numerical systems. After adding in the use of bulk viscosity during plastic flow of the material, we perform two additional simulations to investigate the necessity of artificial viscosity in this regime. Bulk viscosity is used in both 


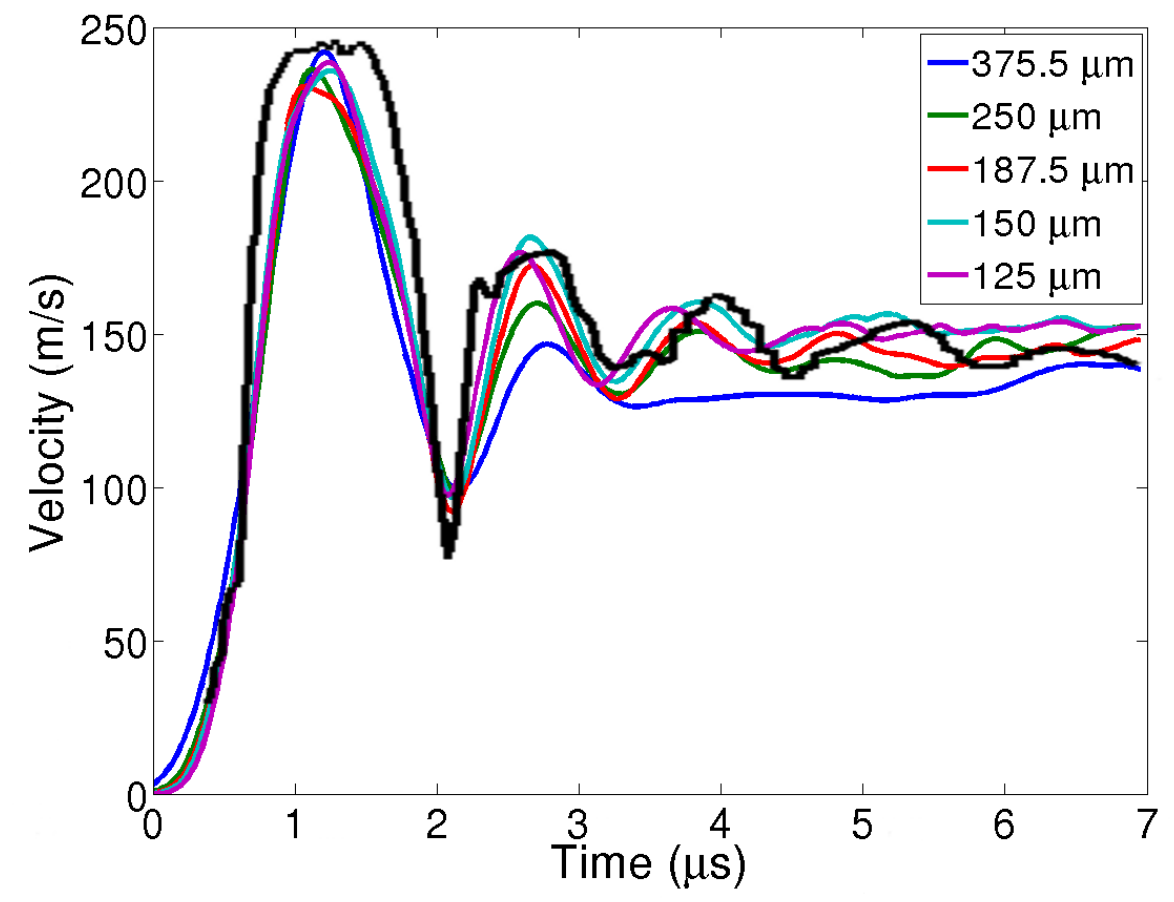

Figure 6: Plot of numerical target plate velocity with time. Simulations are presented using different mesh resolutions, with experimental results in black. 

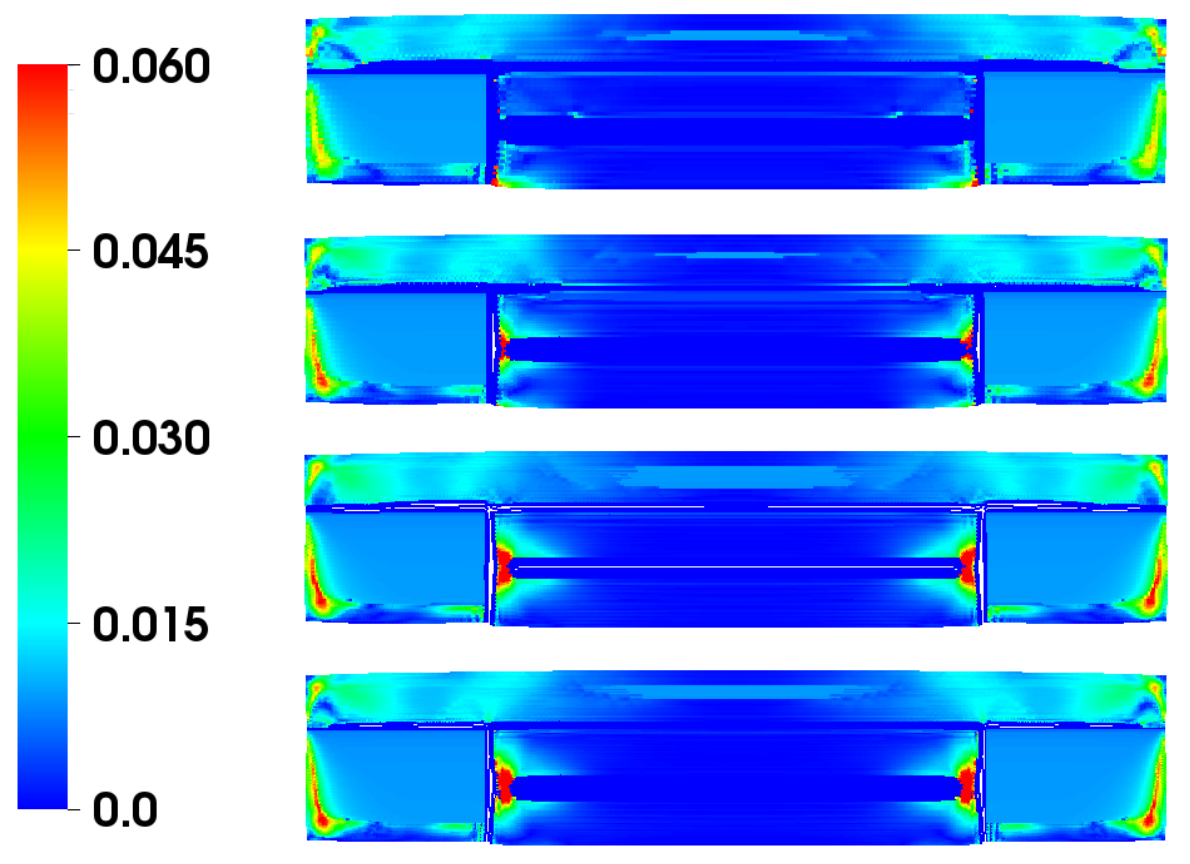

Figure 7: From top to bottom, effective plastic strain calculated using square cells with sizes of $250 \mu \mathrm{m}, 187.5 \mu \mathrm{m}, 150 \mu \mathrm{m}$, and $125 \mu \mathrm{m}$ at $4 \mu \mathrm{s}$ after the impact. 

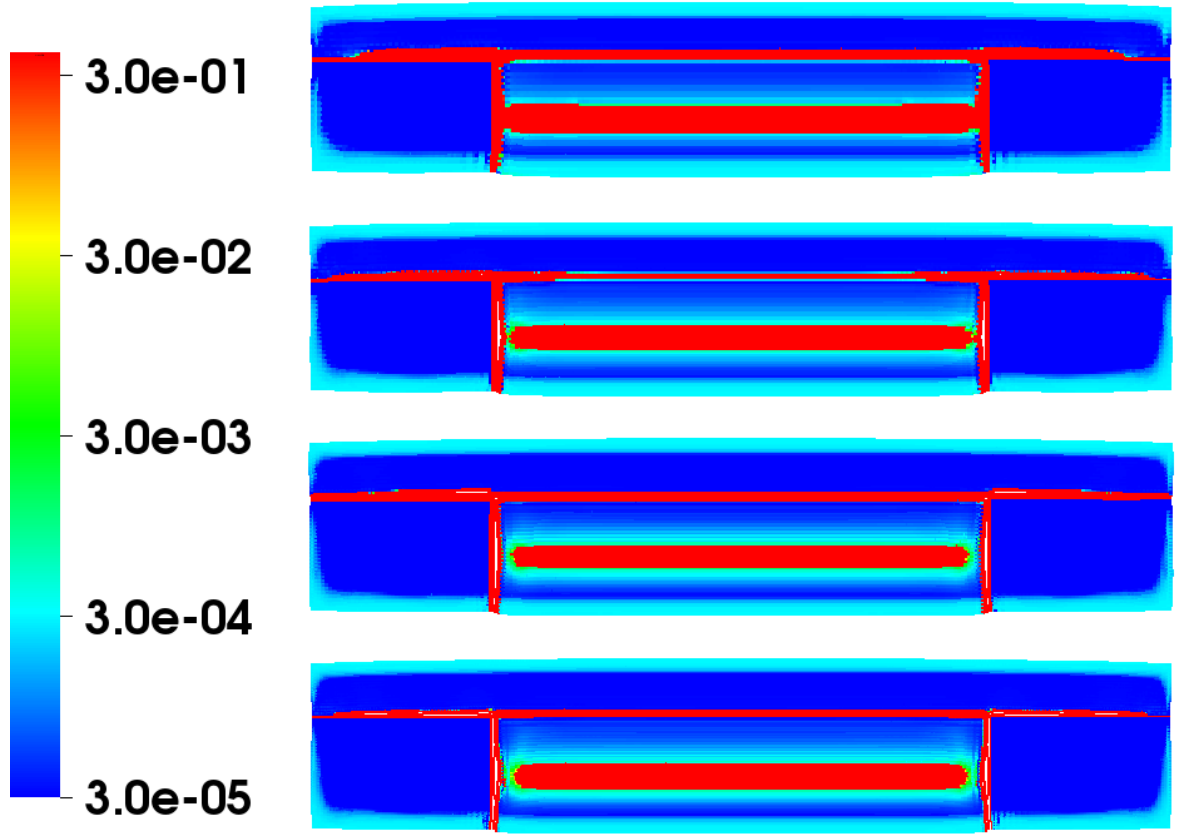

Figure 8: From top to bottom, porosity calculated using square cells with sizes of $250 \mu \mathrm{m}, 187.5$ $\mu \mathrm{m}, 150 \mu \mathrm{m}$, and $125 \mu \mathrm{m}$ at $4 \mu \mathrm{s}$ after the impact. 


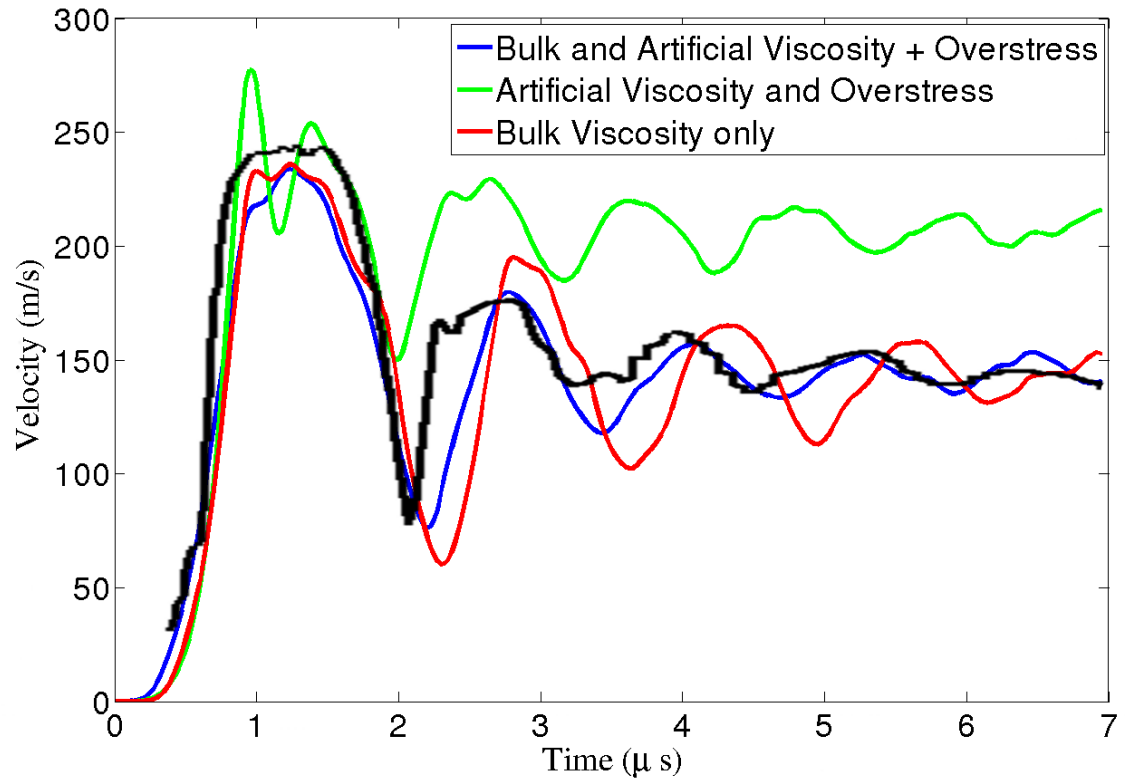

Figure 9: Target plate velocity with and without bulk viscosity present. The problem is simulated using only artificial viscosity and overstress (green), bulk viscosity only (red), and with all three (blue). Experimental results shown in black for comparison. 


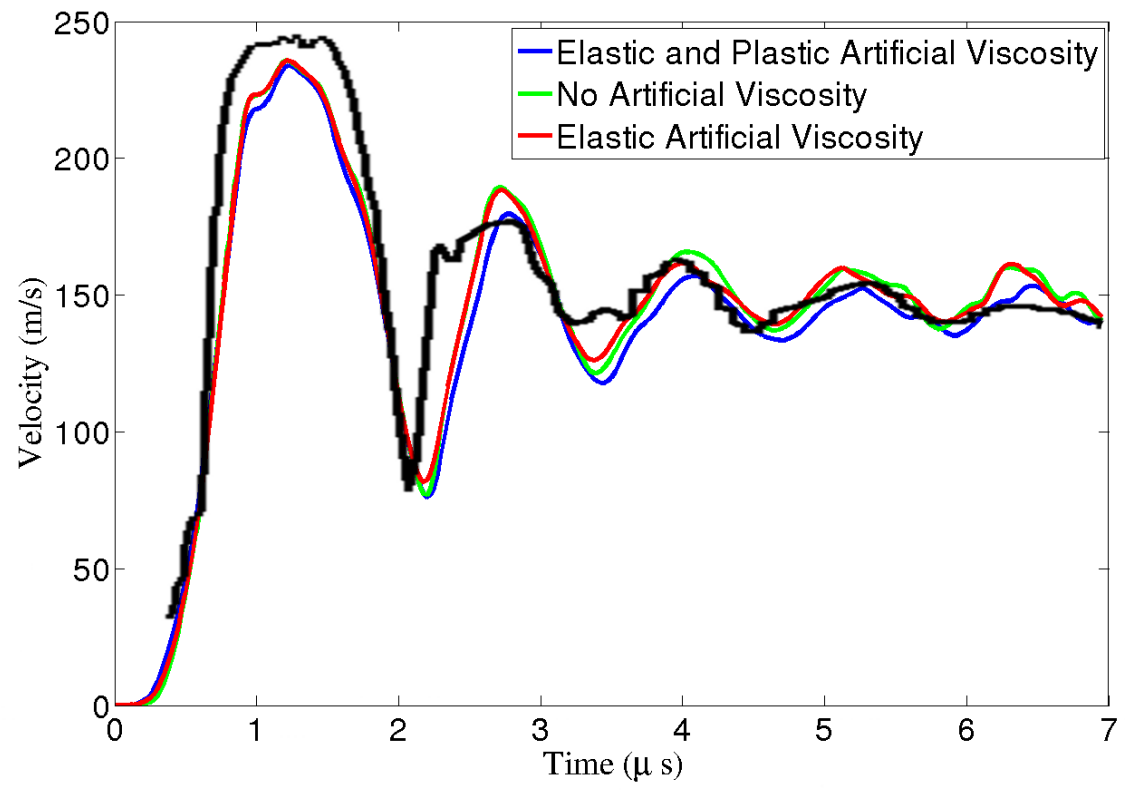

Figure 10: Plot of numerical target plate velocity results using artificial velocity in both elastic and plastic flows (blue), elastic flow only (red), and with no artificial viscosity (green).

and is assumed constant at $10000 \mathrm{~Pa} \cdot \mathrm{s}$ during plastic flow. The artificial viscosity is removed in one simulation and is enabled only during the elastic deformation in the other. The results shown in Fig. 10 demonstrate only a weak dependence of the solution on artificial viscosity, and further suggests that the bulk viscosity term utilized in these simulations is in fact a physical viscosity of the ductile material. It is sufficient in these simulations to consider bulk viscosity and the overstress model without using an artificial viscosity. Although we use a constant value of $10000 \mathrm{~Pa} \cdot \mathrm{s}$, this value may be dependent on the local strain rate of the material. Clearly, physical properties of this bulk viscosity during the plastic flow and its relationship between the strain rate warrant further study.

\subsection{DDMP vs. $M P M$}

Our choice of DDMP in these simulations is predicated on the fact that the material points will move out of their initial cells in the flyer plate simulation. It is expected that the numerical noise described in Sec. 2 will manifest. To confirm this and demonstrate the improvements brought about by DDMP, a simulation was run using traditional MPM, with the same physical and numerical parameters. 


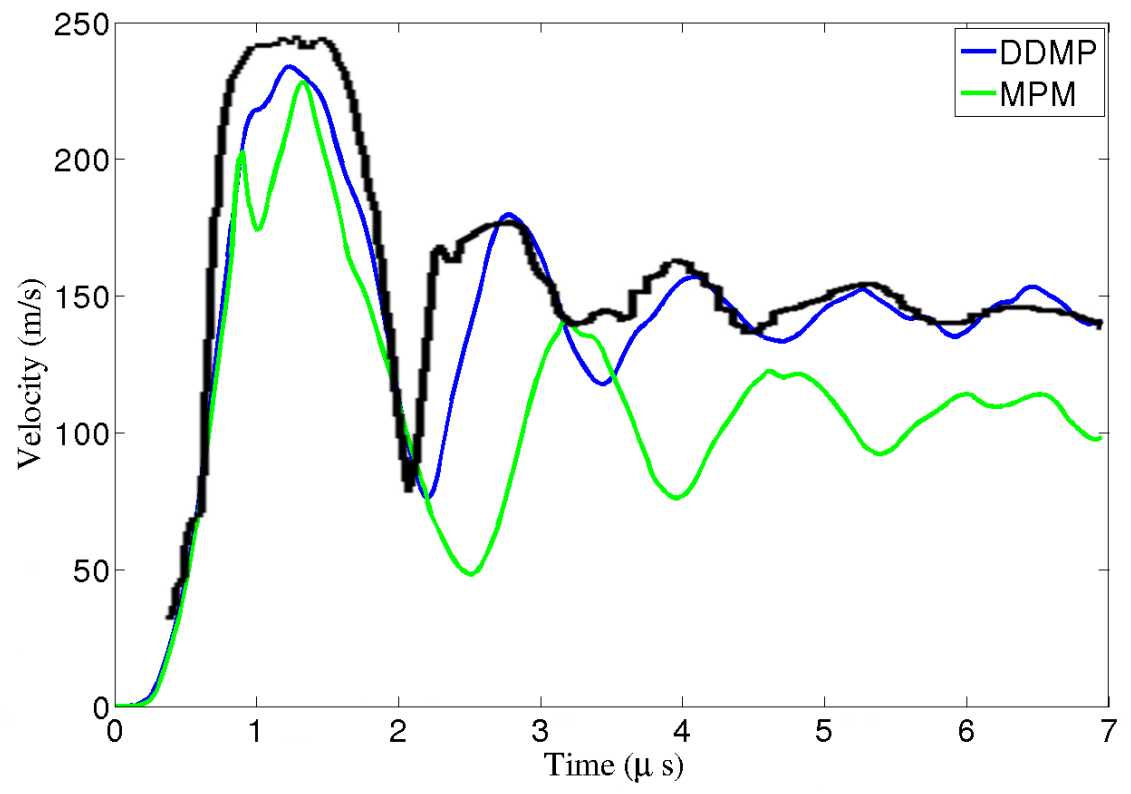

Figure 11: Target plate velocity of simulations run using MPM and DDMP. Experimental data shown in black.

The velocity of the target plate with time is compared to the DDMP result and the experiment in Fig. 11. As can be seen, the velocity difference between the two methods is significant. A stress plot is also shown for each case in Fig. 12. The figure shows the stress field at $1.3 \mu$ s after the impact, when the stress field is critical to accurately project damage to the material. The MPM case demonstrates a large amount of "checker boarding" in the stress field, which is caused by particles moving across cell boundaries, switching the signs on the internal forces. The DDMP method is clearly a dramatic improvement over the MPM in this regard. This improvement comes at the cost of about $45 \%$ more in the amount of computation. For the results shown in Fig. 11, DDMP took about 4 hours, while MPM took about 2 hour and 45 minutes with a single 2.109 GHz AMD Opteron processor in a Linux workstation.

\section{Conclusions}

This work explores the implementation of a material model for ductile damage and failure into a material point method framework. Simulations of a flyer plate 


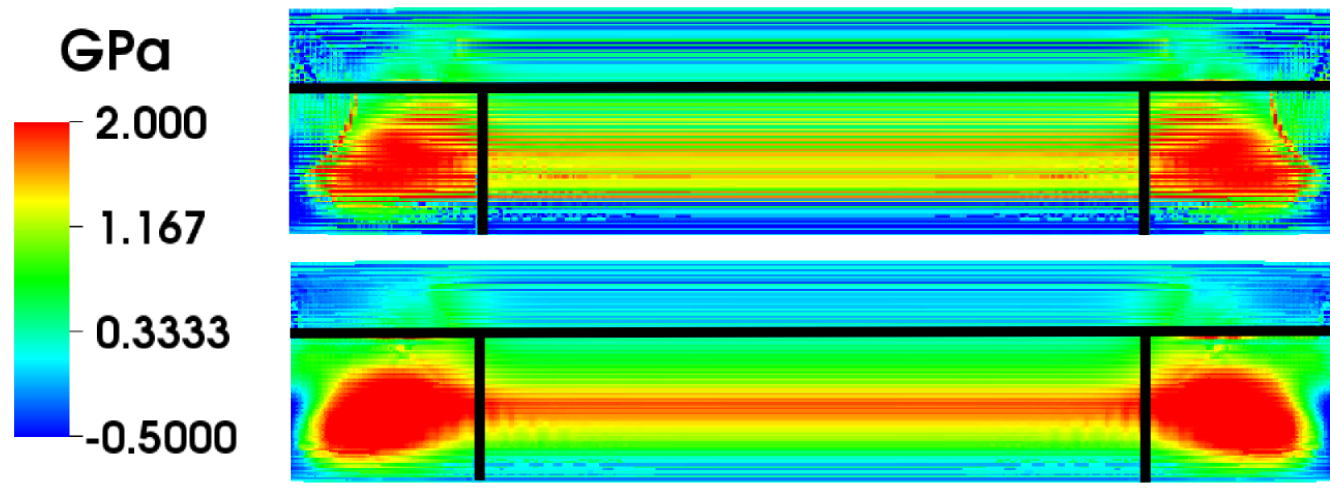

Figure 12: Stress field of $\sigma_{y y}$ from MPM (top) and DDMP (bottom) at $1.3 \mu \mathrm{s}$.

\begin{tabular}{|c|c|c|c|}
\hline Variable & Value & Units & Source \\
\hline$\rho_{s_{0}}$ & 16640 & $\mathrm{~kg} / \mathrm{m}^{3}$ & \\
$G$ & 7.0496 & $\mathrm{GPa}$ & \\
$Y$ & 188.93 & $\mathrm{GPa}$ & \\
$\Gamma_{s}$ & 1.6 & & \\
$A_{1}$ & 185 & $\mathrm{MPa}$ & {$[33]$} \\
$A_{2}$ & 675 & $\mathrm{MPa}$ & {$[33]$} \\
$A_{3}$ & 0.047 & none & {$[33]$} \\
$n$ & 0.3 & none & {$[33]$} \\
$m$ & 0.425 & none & {$[33]$} \\
$Y_{f}$ & 700 & $\mathrm{MPa}$ & \\
$q_{1}$ & 1.5 & none & {$[34]$} \\
$q_{2}$ & 0.625 & none & {$[34]$} \\
$q_{3}$ & 2.25 & none & {$[34]$} \\
$c_{L}$ & 0.5 & none & \\
$c_{q}$ & 2 & none & \\
$T_{m}$ & 3250 & $\mathrm{~K}$ & {$[33]$} \\
\hline
\end{tabular}

Table 1: A list of all material and numerical values used in the simulations, and the source, if applicable. 
problem are carried out using this implementation, and are compared with experimental measurements. While a traditional MPM approach fails due to excessive cell crossing noise, DDMP performs quite well. These results further establish DDMP as a robust, viable method when dealing with problems involving strong history dependence and large material displacement. Further, the material model integrated with this material point approach was shown to perform in a stable fashion.

In our implementation of the ductile material damage and failure model we find that the procedures of solving four nonlinear equations to find the equilibrium state during plastic flow can be simplified to solve only one nonlinear equation for porosity. Since porosity is bounded, a bisection method can be used to guarantee convergence.

By studying the effects of artificial and bulk physical viscosities on the solution, we find that artificial viscosity is not necessary in this problem, while bulk physical viscosity is critical. The physical shear viscosity does not significantly affect the results either because the plastic flow provides sufficient energy dissipation for deviatoric deformation, while the bulk viscosity is the only energy dissipation mechanism for volumetric deformation in the current model. The relationship between high strain rate and the physical viscosity is a subject worth exploring in the future.

\section{Acknowledgments}

The authors gratefully acknowledge the financial support of the DoE/DoD Joint Munitions Program, HE safety program, and many fruitful discussions with Dr. F. L. Addessio and Dr. D. J. Luscher.

\section{References}

[1] D. Z. Zhang, X. Ma, P. T. Giguere, Material point method enhanced by modified gradient of shape function, Journal of Computational Physics 230 (2011) 6379-6398.

[2] D. Sulsky, Z. Chen, H. L. Schreyer, A particle method for history-dependent materials, Computational Methods in Applied Mechanics and Engineering 118 (1994) 179-196.

[3] F. H. Harlow, The particle-in-cell computing method for fluid dynamics, Methods Comput. Phys 3 (1964) 319. 
[4] S. G. Bardenhagen, E. M. Kober, The generalized interpolation material point method, Computer Modeling in Engineering and Sciences 5 (2004) 477-495.

[5] A. Sadeghirad, R. M. Brannon, J. Burghardt, A convected particle domain interpolation technique to extend applicability of the material point method for problems involving massive deformations, International Journal of Numerical Methods in Engineering 86 (2011) 1435-1456.

[6] X. Ma, D. Z. Zhang, P. T. Giguere, C. Liu, Axisymmetric computation of Taylor cylinder impacts of ductile and brittle materials using original and dual domain material point methods, International Journal of Impact Engineering 54 (2013) 96-104.

[7] D. Z. Zhang, B. Jayaraman, Equations and closure models for material pulverization and debris flow, International Journal of Multiphase Flow 56 (2013) 149-159.

[8] J. N. Johnson, F. L. Addessio, Tensile plasticity and ductile failure, Journal of Applied Physics 64 (1988) 6699-6712.

[9] F. L. Addessio, J. N. Johnson, Rate-dependent ductile failure model, Journal of Applied Physics 74 (1993) 1640-1648.

[10] D. Z. Zhang, Q. Zou, W. B. VanderHeyden, X. Ma, Material point method applied to multiphase flows, Journal of Computational Physics 227 (2008) 3159-3173.

[11] S. Ma, X. Zhang, Y. Lian, X. Zhou, Simulation of high explosive explosion using adaptive material point method, Computer Modeling in Engineering and Sciences 2 (2009) 110-123.

[12] B. Jayaraman, X. Ma, P. T. Giguere, D. Z. Zhang, Single-and multi-velocity formulations for impact and pulverization, International Journal of Impact Engineering 49 (2012) 11-21.

[13] Z. T. Ma, X. Zhang, P. Huang, An object-oriented MPM framework for simulation of large deformation and contact of numerous grains, Computer Modeling in Engineering and Sciences 55 (2010) 61-88. 
[14] P. G. Ciarlet, The Finite Element Method for Elliptic Problems, NorthHolland Publishing Company, Amsterdam, 1978.

[15] J. U. Brackbill, H. M. Ruppell, FLIP: a method for adaptively zoned, particle-in-cell calculaions of fluid flows in two dimensions, Journal of Computational Physics 65 (1986) 314-343.

[16] Q. H. Zuo, J. R. Rice, An implicit algorithm for a rate-dependent ductile failure model, Journal of Applied Physics 104 (2008) 083526.

[17] J. N. Johnson, Dynamic fracture and spallataion in ductile solids, Journal of Applied Physics 52 (1981) 2812-2825.

[18] G. R. Johnson, W. H. Cook, Fracture characteristics of three metals subjected to various strains, strain rates, temperatures and pressures, Engineering Fracture Mechanics 21 (1985) 31-48.

[19] A. L. Gurson, Continuum theory of ductile rupture by void nucleation and growth: Part I-Yield criteria and flow rules for porous ductile media, Journal of Engineering Materials and Technology 99 (1977) 2-15.

[20] J. W. Hancock, A. C. Mackenzie, On the mechanisms of ductile failure in high-strength steels subjected to multi-axial stress-states, Journal of the Mechanics and Physics of Solids 24 (1976) 147-160.

[21] J. von Neumann, R. D. Richtmyer, A method for the numerical simulation of hydrodynamics shocks, Journal of Applied Physics 21 (1950) 232.

[22] R. Landshoff, A numerical method for treating fluid flow in the presence of shocks, Tech. Rep. LA-1930, Los Alamos Scientific Laboratory (1955).

[23] M. L. Wilkins, Use of artificial viscosity in multidimensional fluid dynamic calculations, Journal of Computational Physics 36 (1980) 281-303.

[24] J. W. White, A new form of artificial viscosity for elastic solids, Journal of Computational Physics 16 (1974) 119-126.

[25] E. J. Caramana, M. J. Shashkov, P. P. Whalen, Formulations of artificial viscosity for multi-dimensional shock wave computations, Journal of Computational Physics 144 (1998) 70-97. 
[26] T. V. Kolev, R. N. Rieben, A tensor artificial viscosity using a finite element approach, Journal of Computational Physics 228 (2009) 8336-8366.

[27] J. M. Owen, A tensor artificial viscosity for SPH, Journal of Computational Physics 201 (2004) 601-629.

[28] V. A. Dobrev, T. E. Ellis, T. V. Kolev, R. N. Rieben, High-order curvilinear finite elements for axisymmetric Lagrangian hydrodynamics, Computer and Fluids 83 (2013) 58-69.

[29] Y. Bazilevs, C. C. Long, I. Akkerman, D. J. Benson, M. J. Shashkov, Isogeometric analysis of lagrangian hydrodynamics: Axisymmetric formulation in the rz-cylindrical coordinates, Journal of Computational Physics 262 (2014) 244-261.

[30] V. N. Mineev, A. V. Mineev, Viscosity of metals under shock-loading conditions, Journal de Physique IV 7 (1997) 583-585.

[31] D. Z. Zhang, W. B. VanderHeyden, Q. Zou, N. T. Padial-Collins, Pressure calculations in disperse and continuous multiphase flows, Journal of Multiphase Flow 33 (2007) 86-100.

[32] J. Millet, G. Whiteman, N. Bourne, S. Case, R. Gray, Shear strength development in tantalum alloys: Effects of cold work and alloying, in: Bulletin of the American Physical Society, Vol. 58, Seattle, Washington, 2013, pp. 883-887.

[33] S. R. Chen, G. T. G. III, Constitutive behavior of tantalum and tantalumtungsten alloys, Metallurgical and Materials Transactions 27 (A) (1996) 2994-3006.

[34] C. A. Bronkhorst, E. K. Cerreta, Q. Xue, P. J. Maudlin, T. A. Mason, G. T. G. III, An experimental and numerical study of the localization behavior of tantalum and stainless steel, International Journal of Plasticity 22 (2006) 13041335 . 